\title{
Effects of Task Difficulty and Target Likelihood in Area V4 of Macaque Monkeys
}

C. Elizabeth Boudreau, Tori H. Williford and John H. R. Maunsell

J Neurophysiol 96:2377-2387, 2006. First published Jul 19, 2006; doi:10.1152/jn.01072.2005

You might find this additional information useful...

This article cites 39 articles, 13 of which you can access free at:

http://jn.physiology.org/cgi/content/full/96/5/2377\#BIBL

Updated information and services including high-resolution figures, can be found at:

http://jn.physiology.org/cgi/content/full/96/5/2377

Additional material and information about Journal of Neurophysiology can be found at: http://www.the-aps.org/publications/jn

This information is current as of October 18, 2006 . 


\title{
Effects of Task Difficulty and Target Likelihood in Area V4 of Macaque Monkeys
}

\author{
C. Elizabeth Boudreau, ${ }^{2}$ Tori H. Williford, ${ }^{1,2}$ and John H. R. Maunsell ${ }^{1,2}$ \\ ${ }^{1}$ Howard Hughes Medical Institute and ${ }^{2}$ Department of Neuroscience, Baylor College of Medicine, Houston, Texas
}

Submitted 11 October 2005; accepted in final form 12 July 2006

\begin{abstract}
Boudreau, C. Elizabeth, Tori H. Williford, and John H. R. Maunsell. Effects of task difficulty and target likelihood in area V4 of macaque monkeys. J Neurophysiol 96: 2377-2387, 2006. First published July 19, 2006; doi:10.1152/jn.01072.2005. Spatial attention improves performance at attended locations and correspondingly modulates firing rates of cortical neurons. The size of these behavioral and neuronal effects depends on the difficulty of the task performed at the attended location. Psychological theorists have attributed this to a tighter focus of a fixed amount of processing resource at the attended location, but the effects of task difficulty on the distribution of neuronal effects of attention across the visual field have not been fully explored. We trained rhesus monkeys to do a detection task in which difficulty and spatial attention were manipulated independently. Probe stimuli were used to measure behavioral performance in different conditions of attention and difficulty. Animals performed better at attended locations and this advantage increased with difficulty, consistent with data from human psychophysics. Neuronal modulation by spatial attention was larger with greater difficulty. In two animals, increasing difficulty caused a modest increase in neuronal responses to visual stimuli regardless of the locus of spatial attention. In a third animal, which was previously trained to ignore multiple distracting stimuli, increasing task difficulty increased responses at the focus of attention and suppressed responses away from the focus of attention. The results show that difficulty can modulate effects of spatial attention in V4; it can alter the distribution of sensory responses across the visual scene in ways that may depend on the subject's behavioral strategy.
\end{abstract}

\section{N T R O D U C T I O N}

Human psychophysical studies have demonstrated that attentional load, defined as either the number of relevant items or the complexity of the processing required for the task, can have a profound impact on behavioral effects of spatial attention (Lavie and Tsal 1994; Sade and Spitzer 1998; Urbach and Spitzer 1995). For example, orientation-discrimination thresholds for peripherally presented stimuli are elevated when subjects perform a demanding task at fixation, as opposed to merely fixating (Lee et al. 1997). Increasing the difficulty of a central task reduces interference from peripheral distractors (LaBerge et al. 1991; Lavie and Cox 1997).

Because high attentional load can reduce interference from distractors outside the attentional focus, it has been proposed that spatial attention has a fixed capacity and that increasing load at an attended location concentrates the fixed amount of attentional resource at that location, necessarily withdrawing it elsewhere (Lavie 1995). This attentional load hypothesis accounts for increases in behavioral performance at the focus of

Address for reprint requests and other correspondence: J. Maunsell, Harvard Medical School, Department of Neurobiology, 220 Longwood Avenue, Boston, MA 02115 (E-mail: maunsell@hms.harvard.edu). attention, as more attentional resource is directed there. In addition, it dictates a decline in performance away from the attentional focus as the resource is removed. What neurophysiological changes correspond to the behavioral effects of increased load?

Several single-units studies have shown that increasing task difficulty can affect the strength of neuronal responses to visual stimuli. The visual responses of neurons in monkey prefrontal cortex have shown to be modulated by increased difficulty associated with discriminating stimuli that are more similar (Bichot et al. 2001), locating targets embedded in more distractors (Bichot et al. 2001), or tasks involving greater memory load (Hasegawa et al. 2004). In visual cortex, Spitzer and colleagues (Spitzer and Richmond 1991; Spitzer et al. 1988) showed that increasing task demands for a single relevant stimulus increased neuronal responses to that stimulus. Motter (1993) found that attentional modulations were more common in V4 neurons when many distractor stimuli were added to the stimulus display. In humans, increasing attentional load in an unrelated task decreases the strength of a motion aftereffect and functional magnetic resonance imaging (fMRI) signals associated with moving stimuli in human MT/V5 (Rees et al. 1997).

However, the effect of increasing attentional load at one location on neuronal responses to stimuli at distant sites is not well studied. Changes at distant sites are important for evaluating whether spatial attention has a fixed capacity. If the mechanism of spatial attentional modulation of neuronal responses acts as a fixed resource, then responses to the distant stimulus should be suppressed as load is increased elsewhere. Studies of human attention, however, suggest that increasing the difficulty of attentional tasks may increase processing efficiency in a nonspatially specific manner, by increasing vigilance (Parasuraman et al. 1998). Such vigilance increases might cause neuronal responses to a distant stimulus to increase. A better understanding of how behavioral modulations of neuronal responses are distributed across the visual field is important for understanding how such modulations help specific stimuli for enhanced processing.

We examined this question by recording the responses of individual neurons in area $\mathrm{V} 4$ of macaque monkeys performing a task in which the spatial location of targets and task difficulty were controlled independently. The results suggest that mechanism related to spatial-directed attention and overall vigilance interact to determine neuronal responses to distant stimuli, and that the net effect depends on their relative contributions.

The costs of publication of this article were defrayed in part by the payment of page charges. The article must therefore be hereby marked "advertisement" in accordance with 18 U.S.C. Section 1734 solely to indicate this fact. 
Preliminary results from this experiment were previously reported (Boudreau and Maunsell 2001).

\section{METHODS}

\section{Animals and surgery}

The animal protocols used in this study were approved by the Baylor College of Medicine Institutional Animal Care and Use Committee. Three male rhesus monkeys (Macaca mulatta, 5-7 kg) served as subjects in these experiments. Each animal underwent 2-4 mo of training on the task used in this experiment before recording began. Details of the training histories of the animals are included in the RESULTS and DISCUSSION sections.

After training, a recording chamber (20 mm diameter) was placed over area V4 in the right hemisphere. Animals had restricted access to water during training and recording and their behavior was shaped using operant conditioning with positive reinforcement only. They worked $1-5 \mathrm{~h}$ each day for juice rewards.

\section{Eye position monitoring}

A headpost and scleral search coil were implanted in an initial aseptic surgery (Judge et al. 1980). Horizontal and vertical positions of the right eye were monitored with the search coil (Robinson 1963) and sampled every 2 or $5 \mathrm{~ms}$ [root-mean-square (RMS) noise about $0.03^{\circ}$ ]. The calibration of eye position locations was continuous during data collection, with each trial using one of four possible fixation positions chosen pseudorandomly.

Animals were required to maintain eye position within a small window $\left( \pm 0.65-1.3^{\circ}\right)$ around a fixation point. Trials were aborted and the animals received no reward if their gazes left this window at any time during the trial. Some analyses were based on small saccades that occurred within the bounds of the fixation window. These were detected off-line using speed and duration criteria. The occurrence of a saccade was defined by a minimum 10-ms interval (three or six samples) during which the instantaneous speed of the eye was $>15 \%$, with $<45^{\circ}$ deviation in direction between successive samples. Saccade ends were assigned when the instantaneous speed fell to $<15^{\circ} \mathrm{s}$ for $10 \mathrm{~ms}$. Inspection of eye position traces suggested that these criteria reliably detected displacements $>0.25^{\circ}$.

\section{Stimulus presentation}

Stimuli were presented on a calibrated CRT video display (screen width: 36 or $39 \mathrm{~cm} ; 75 \mathrm{~Hz} ; 1,600 \times 1,200$ pixels) positioned $58 \mathrm{~cm}$ from the animal. The average screen luminance was $23 \mathrm{~cd} / \mathrm{m}^{2}$. No other visible light sources were in the recording room. Stimuli were achromatic $100 \%$ contrast Gabor patches with the same average luminance as the background, temporally counterphased in contrast with a 4-Hz sinusoid.

Gabor stimuli were adjusted in location, size, spatial frequency, and orientation to best drive each neuron isolated for data collection, as assessed by listening to spikes on an audio monitor. Receptive field centers ranged from 0.7 to $5.6^{\circ}$ in eccentricity (medians: $1.5^{\circ}$ for Animal $1 ; 3.8^{\circ}$ for Animal 2; $2.9^{\circ}$ for Animal 3). SD values of the Gabors used ranged from 0.2 to $1.5^{\circ}$ (medians: $0.4^{\circ}$ for Animal $1 ; 0.5^{\circ}$ for Animal 2; $0.7^{\circ}$ for Animal 3). Spatial frequencies were between 0.24 and 6.0 cycles/deg (medians: 2.7 cycles/deg for Animal 1; 1.0 cycle/deg for Animal 2; 3.2 cycles/deg for Animal 3). Four neurons did not have obvious orientation selectivity during data collection; data were collected from these neurons after assigning an arbitrary preferred orientation. These neurons were not distinct in other ways.

\section{Behavioral task}

Animals did an orientation change detection task using Gabor patches (Fig. 1A). At the beginning of each trial, the animals were

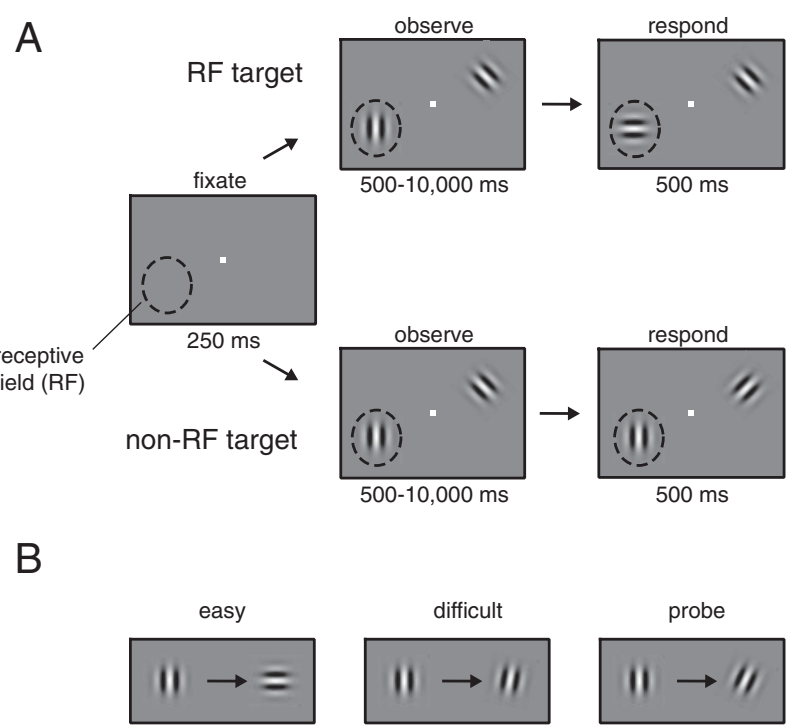

FIG. 1. Behavioral task. A: each trial began with fixation on a small spot at the center of the screen. Animals were then presented with counterphasing Gabor stimuli at 2 spatial locations. After a variable period, one of the stimuli would change orientation. Animal responded to this change either by releasing a lever (Animals 1 and 3) or making a saccade toward the changed stimulus (Animal 2). B: task difficulty was controlled by changing the size of the orientation change. In easy blocks of trials, the orientation change was $90^{\circ}$. In difficult blocks, it was set to a challenging size. Randomly inserted probe trials had orientation changes that were intermediate. These were used to assess whether the animal's performance was affected by the likely location and task difficulty.

required to press and hold down a lever (Animals 1 and 3 only) and to acquire and maintain fixation on a white square $\left(0.25^{\circ}\right.$ width $)$ presented near the center of the screen. Two Gabor patches appeared on the screen during each trial. One, presented within the receptive field of a neuron under study, was oriented either parallel to or orthogonal to the estimated preferred orientation of the cell. A second Gabor, rotated by $\pm 45^{\circ}$ relative to the receptive field Gabor but otherwise identical, was presented in a position diametrically opposed across the fixation spot. Thus whereas the receptive field stimulus was always either the preferred orientation or $90^{\circ}$ from preferred, the stimulus in the opposite hemifield was always $\pm 45^{\circ}$ from the preferred orientation. The Gabor was offset in orientation so that the task could not be solved by detecting small differences in orientation between the two stimuli. The animals were trained to maintain fixation while monitoring the Gabor patches for a change in orientation.

The animals were trained to respond when either Gabor changed orientation by either releasing a lever (Animals 1 and 3) or making a saccade to the changed Gabor (Animal 2) within $600 \mathrm{~ms}$ to signal that they had detected the change; for this they received a reward. A high tone was played as auditory feedback for a correct response. If they failed to maintain fixation or responded $<100 \mathrm{~ms}$ after the change, the trial was aborted immediately (with a low tone to indicate an incorrect response) and no reward was given. Also, if they failed to respond to the correct target within the specified time window after a change occurred (missed trial), the low tone was played and no reward was given.

Orientation changes were restricted to occur when the contrast of the counterphasing Gabor crossed zero, which happened eight times per second. Across trials, the times of the orientation change were exponentially distributed with a median of about $2 \mathrm{~s}$ and a minimum of $500 \mathrm{~ms}$. If no change had occurred after $10 \mathrm{~s}$ ( $8 \mathrm{~s}$ for Animal 2), the trial was ended automatically and counted as correct. To encourage the animals to wait for later orientation changes, the size of the reward increased with time elapsed before the orientation change. 
Trials were performed in blocks of four different types that were pseudorandomly interleaved. Blocks were defined by task difficulty and spatial location of attention. Each block was either 16 or 32 trials long, and at least two repetitions of each block type were collected for all neurons included in the analysis.

To bias the spatial location of attention, in each block one of the two Gabors was much more likely ( $87.5 \%$ of trials) to change in orientation (Fig. 1A, Table 1). The animals' attention was directed toward the likely target using instruction trials, in which only one Gabor appeared, at the beginning of the block. On a subset of behavioral experiments, a third attentional condition was used, in which instruction trials cued both Gabor locations at the beginning of each block. In these trials, the animal had no information about which stimulus was more likely to change in orientation (and both were equally likely).

Task difficulty was manipulated by varying the size of the orientation change that the animals had to detect at the likely location (Fig. $1 B$ ). Trials were performed at two levels of difficulty (referred to here as difficult and easy). The orientation changes in easy blocks were $90^{\circ}$. The size of the orientation change in the difficult blocks was adjusted for each stimulus configuration to maintain the animals' average performance on difficult trials within a target range. The orientation changes in difficult blocks were between 5 and $9^{\circ}$ for Animal 1, between 4 and $15^{\circ}$ for Animal 2, and between 8 and $14^{\circ}$ for Animal 3. The size of the change used during data collection for each neuron was fixed. Orientation changes at the unlikely location were always "probe trials," described below, for which the size of the orientation change was held constant at a value intermediate to the easy and difficult values.

Because the animals were required to compare only the orientation of the stimulus after the change to its orientation immediately before, there was little demand on working memory, compared with other tasks, such as match-to-sample. This allowed us to separate increases in attentional load arising from increased task difficulty from increases in memory load (Rees et al. 1997). Also, this task required the animals to monitor stimuli over a period of time for infrequent and unpredictable targets. This type of task is thought to draw on the intensive dimension of attention, as opposed to the spatial dimension of attention (Parasuraman 1984), so that changes in difficulty were likely to change the amount of attention devoted to the task.

\section{Assessment of behavior}

Although the animals were encouraged to direct their attention to a particular spatial location by biasing target likelihood, and were encouraged to change the amount of effort they devoted to the task by changing task difficulty, we needed a behavioral measure that would show whether these manipulations succeeded in changing the ani-

TABLE 1. Distribution of orientation changes in easy and difficult blocks

\begin{tabular}{lcclcc}
\hline \hline & \multicolumn{2}{c}{ Easy Block } & & Difficult Block \\
\cline { 2 - 3 } \cline { 5 - 6 } & $\begin{array}{c}\text { Orientation } \\
\text { change }\end{array}$ & Frequency & & $\begin{array}{c}\text { Orientation } \\
\text { change }\end{array}$ & Frequency \\
\hline $\begin{array}{l}\text { Likely location regular } \\
\text { Likely location probe }\end{array}$ & $90^{\circ}$ & $75.0 \%$ & & $\sim 8^{\circ}$ & $75.0 \%$ \\
Unlikely location probe & $\sim 10^{\circ}$ & $12.5 \%$ & & $\sim 10^{\circ}$ & $12.5 \%$ \\
\hline
\end{tabular}

Easy blocks consisted of mostly $90^{\circ}$ changes, whereas in difficult blocks most of the changes were $<10^{\circ}$. In each block, one location was more likely ( $87.5 \%$ of trials) to contain the change. Behavior was assessed with probe trials that were similar in difficulty to the regular trials of the difficult blocks and occurred with equal frequency at both spatial locations and in both easy and difficult blocks. Animals were instructed as to the identity of the likely location at the beginning of each new block. mals' behavior. To measure the ability of the animals to respond to orientation changes as a function of target likelihood and block difficulty, we used infrequent and randomly inserted probe trials that occurred equally often at both likely and unlikely locations, and equally often in difficult and easy blocks ( $25 \%$ of all trials). The size of the orientation change on probe trials was usually intermediate to the difficult and easy changes (Fig. $1 B, 7-20^{\circ}$ ) and did not vary between easy and difficult blocks for a given cell. For Animal 1, the orientation change on probe trials was sometimes set equal to or smaller than the difficult changes to prevent saturation of performance on probe trials. Because the stimulus was the same for probe trials in all conditions, differences in the animals' abilities to detect the orientation change on probe trials could be attributed to changes internal to the animals related to the locus of attention or the difficulty of the block (Spitzer et al. 1988).

\section{Neurophysiological data collection}

We made small craniotomies over V4 while the animals were anesthetized with $15 \mathrm{mg} / \mathrm{kg}$ ketamine, leaving the dura intact. A new craniotomy was made approximately every $3 \mathrm{wk}$. In daily recording sessions, transdural $\mathrm{Pt} / \mathrm{Ir}$ electrodes $(0.8-2.2 \mathrm{M} \Omega$ at $1 \mathrm{kHz})$ were positioned using an $\mathrm{X}-\mathrm{Y}$ stage. They were advanced through the dura by a hydraulic microdrive. After the stage and microdrive were attached to the chamber each day, the chamber was filled with sterile mineral oil to reduce pulsations. Animals received two to four craniotomies, with one to 13 penetrations made in each craniotomy.

Recordings were made in gray matter within $4 \mathrm{~mm}$ from the cortical surface. Signals from the microelectrodes were filtered, amplified, and sent to an oscilloscope and audio monitor. The animals performed the task as described above while the electrode was advanced through cortex. After a unit was isolated, the task was changed briefly (about $5 \mathrm{~min}$ ) to a simple fixation task while stimuli were optimized for the cell. Individual units were isolated using a window discriminator and spike times were recorded with 1-ms resolution by the same computer that controlled and monitored the behavioral task. Time bases were synchronized with the vertical retrace of the video display.

\section{Neurophysiological data analysis}

For the assessment of the effects of spatial attention and task difficulty on neuronal responses, data were analyzed from the first time an orientation change could have occurred (500 ms after stimulus onset) up to the time at which the orientation actually changed on that trial, yielding a variable amount of data per trial. We recorded data from $\geq 32$ trials in each of the four behavioral conditions (2 likely locations $\times 2$ block difficulties). Spontaneous firing rates were computed from a 250 -ms period in each trial after the animal had achieved fixation but before the stimulus appeared. Only correct trials were included in these analyses.

All tests of statistical significance were computed relative to a 0.05 false positive rate, unless otherwise specified. Statistical tests were performed using Matlab Toolbox functions, where available, and computed according to Zar (1999) otherwise.

\section{RES U L T S \\ Behavioral performance}

After several months of training, each animal developed a stable pattern of behavior. All animals performed substantially better on easy trials than on difficult trials (Animal 1: easy 99.5\% correct, difficult $89.7 \%$; Animal 2: easy $98.3 \%$, difficult $70.3 \%$; Animal 3: easy $94.0 \%$, difficult $86.0 \%$ ). Behavioral performance on probe trials that were presented pseudoran- 
domly and infrequently in all behavioral conditions is presented in Fig. 2.

Difficulty and target likelihood both affected performance on probe trials. These effects were robust, indicating that the animals were motivated to perform and that they were well trained on the task by the time recording began. SE values of the binomial statistic are smaller than the points in Fig. 2. Regardless of the difficulty of the block in which probes occurred, the animals were more likely to correctly detect orientation changes at the likely location than they were to detect the same orientation changes when they occurred at an unlikely location. The differences in behavior arising from target likelihood were significant for all animals in both easy and difficult blocks by $\chi^{2}$ test (all $P<0.001$ ). This demonstrates that the animals used target likelihood to direct their spatial attention to the appropriate stimulus, so that the unlikely stimulus was less attended.

The difficulty of the block in which a probe trial occurred also affected the animals' abilities to detect the orientation change. Equivalent orientation changes on probe trials were more readily detected at the likely location when the animals were working on a block of difficult trials, compared with when the probe appeared amid easy trials. Changes on probe trials at the unlikely location, however, were more poorly detected when they were embedded in a difficult block of trials. The effect of difficulty on behavior was also significant for all animals at both likely and unlikely target locations (all $P<$ 0.001). Because the frequency of occurrence and physical appearance of the probe trials were the same regardless of the difficulty of the block in which they occurred, differences in the animals' abilities to detect orientation changes on these trials reflect the animals' responses to differences in the attentional load of the task.

\section{Modulation of neuronal responses by target likelihood and difficulty}

We examined the effect of task difficulty on the responses of $128 \mathrm{~V} 4$ neurons to stimuli presented at likely and unlikely target locations (51 in Animal 1, 33 in Animal 2, and 44 in Animal 3). For two cells in Animal 2, sufficient data were collected in only two or three of the four behavioral conditions. Although all three animals showed the same pattern of behavioral effects for task difficulty and target likelihood (Fig. 2), neuronal responses differed between animals. Whereas the neuronal and behavioral responses of Animals 1 and 2 were of one type, those from Animal 3 had important differences. In the sections that follow, we first present data from Animals 1 and 2, then data from Animal 3, and finally measurements made to explain the differences between animals. The behav- ioral and physiological effects were similar for Animals 1 and 2 and their data were combined in the subsequent analyses.

Figure 3, $A$ and $B$ shows responses of a neuron from Animal 2 to its preferred orientation during the trial period before an orientation change occurred. Responses are cycle averaged, so that the histogram shows the average activity during one cycle of the 4-Hz temporally counterphasing stimulus $(250 \mathrm{~ms})$. The response of this neuron was strongly affected by both target likelihood and task difficulty. Target likelihood modulated the response in both difficult and easy blocks. Mean driven rates were, respectively, $12.2 \pm 1.3$ and $6.4 \pm 1.0$ spikes/s for likely and unlikely stimuli in the easy blocks and $20.5 \pm 1.7$ and $9.5 \pm 0.9$ spikes/s in the difficult blocks. At both difficulty levels, the driven rates increase substantially when the stimulus was a likely behavioral target (easy blocks 90\% increase; difficult blocks $115 \%$ increase), indicating that the effect of target likelihood was largely independent of difficulty. Responses to both likely and unlikely targets were larger when the task was more difficult (likely targets 68\% increase; unlikely targets $48 \%$ increase).

Most neurons from Animals 1 and 2 were significantly modulated by at least one of the two behavioral manipulations (task difficulty or target likelihood). A repeated-measures ANOVA revealed significant main effects of both task difficulty and target likelihood among the neurons from Animals 1 and $2\left(\mathrm{~F}=33.2, P<10^{-6} ; \mathrm{F}=7.6, P=0.007\right)$, and an interaction between difficulty and likelihood $(\mathrm{F}=16.7, P<$ $10^{-4}$ ). Figure 3, $C$ and $D$ shows the average responses from all neurons in Animals 1 and 2 that were individually significantly modulated by target likelihood in either the easy or difficult trials ( $n=36,43 \%$ of neurons; $t$-test, $P<0.05$ with Bonferroni correction for multiple comparisons). The mean driven rate for these neurons in easy trials was 18.9 spikes/s for the likely location and 18.0 spikes/s for the unlikely location. Responses at both locations were enhanced in the difficult condition, with a mean driven rate of 22.3 spikes/s for the likely location and 19.2 spikes/s for the unlikely location.

Although we focus here on responses to each neuron's preferred orientation, a similar pattern of results was found for responses to the nonpreferred orientation. Average responses to the orientation orthogonal to the preferred orientation were about half as strong, so effects did not reach the same level of statistical significance. A repeated-measures ANOVA found a significant main effect for task difficulty $\left(\mathrm{F}=15.3, P<10^{-3}\right)$ but not target likelihood ( $\mathrm{F}=3.1, P=0.08)$. There was also a significant interaction between difficulty and likelihood for the nonpreferred orientations $(\mathrm{F}=6.2, P=0.015)$. For neurons with statistically significant effects of attention, the average driven rate for the nonpreferred orientation in easy

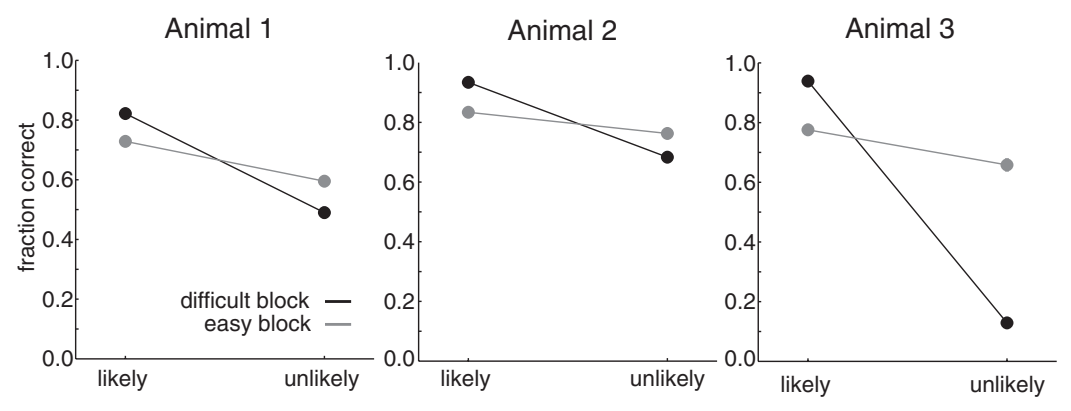

FIG. 2. Behavioral performance. For each animal, plots show the average fraction of correct responses to probe trials of constant difficulty embedded in easy (gray plots) or difficult (black plots) blocks. Responses to probe trials were assessed at 2 spatial locations, one where a change was likely to occur and another where the change was unlikely to occur. SE bars are smaller than the points (Animal 1, $n=1,069$; Animal 2, $n=$ 1,016; Animal 3, $n=755$ ). 
A

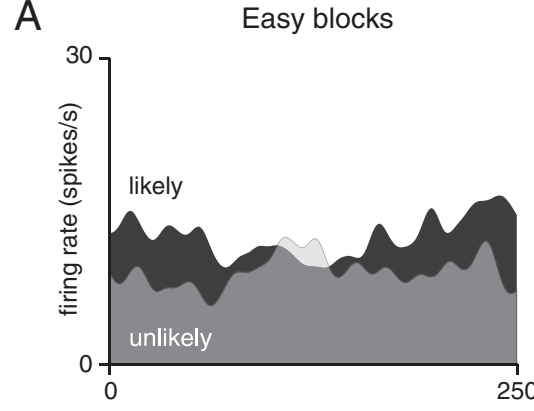

C

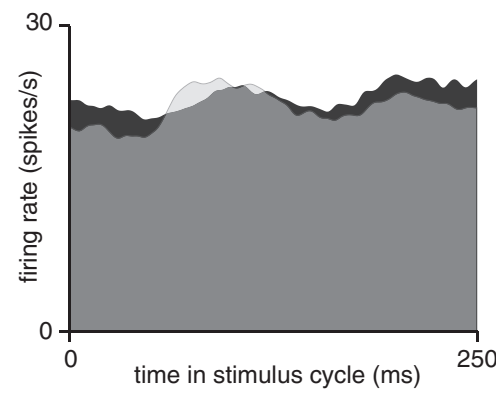

B
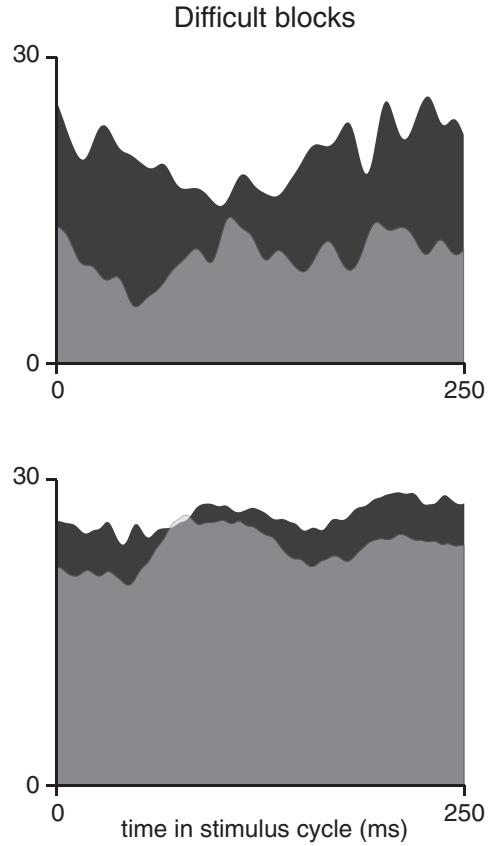

FIG. 3. Effects of load and likelihood on neuronal responses in Animals 1 and 2. $A$ and $B$ : response histograms from an example cell presented with its preferred orientation. A: average responses of the neuron during each counterphase cycle of a stimulus at the likely location (dark gray; average driven rate 12.2 spikes/s) and unlikely location (light gray; average driven rate 6.4 spikes/s) during easy trials. $B$ : average responses to the same stimulus at the likely location (average driven rate 20.5 spikes/s) and unlikely location (average driven rate 9.5 spikes/s) during difficult trials. This neuron responded significantly more to its preferred stimulus when it was likely that the stimulus would change orientation (dark gray histograms) compared with when it was unlikely (light gray histograms). Effect was stronger when the task was difficult $(B)$ than when it was easy (A). $C$ and $D$ : population response histograms from the 36 neurons in Animals 1 and 2 that were significantly modulated by attention. In the easy trials the average driven rate was 18.9 spikes/s for stimuli at the likely location and 18.0 spikes/s for stimuli at the unlikely location. In difficult trials the average response was 22.3 spikes/s for the likely location and 19.2 spikes/s for the unlikely location. Each histogram has been smoothed with a Gaussian filter ( $\sigma=5 \mathrm{~ms}$ for $A$ and $B ; \sigma=3$ ms for $C$ and $D$ ).

trials was 6.6 spike/s for the likely location and 5.9 spike/s for the unlikely location. Responses to the nonpreferred orientation at both locations were enhanced in the difficult condition, with a mean driven rate of 7.5 spikes/s for the likely location and 6.3 spikes/s for the unlikely location.

To quantify the distribution of the size of the effects of difficulty and target likelihood, we computed a target-likelihood ratio for each block difficulty and a difficulty ratio for each target likelihood, on a cell-by-cell basis for responses to the preferred orientation. Figure $4, A$ and $B$ shows the distributions of the target-likelihood ratios, sorted by block difficulty. For Animals 1 and 2, 29/83 and 27/83 neurons showed significant modulation by target likelihood in the difficult and easy blocks, respectively. The median likelihood ratio for all neurons was 1.07 in the difficult blocks and 1.03 in the easy blocks. These effects of target likelihood are smaller than those previously reported for attentional modulation in area V4 (e.g., McAdams and Maunsell 1999), which may reflect the animals' attempts to attend simultaneously to both stimuli.

Figure 4, $C$ and $D$ shows the distributions of the difficulty ratios for responses to likely and unlikely targets. Significant changes in responses to a likely target with increased task difficulty were seen in 40/82 cells and the median ratio was 1.13. Contrary to our expectations and the behavioral results that showed a decline in performance on unlikely targets in the difficult blocks (Fig. 2), neuronal responses to stimuli in the unlikely location increased in most cells as the task demands increased (Fig. 4D). Significant effects of task difficulty on responses to unlikely targets were seen in 21/83 cells. Only four of these showed significant decreases and the median ratio for our sample was 1.06 .

We examined whether the interaction between target likelihood and task difficulty could be explained as a multiplicative scaling by performing a repeated-measures ANOVA on the log transform of the firing rates. Because multiplicative effects are additive in log space, the logarithms of the data should exhibit additivity. We found that there was still a small but significant
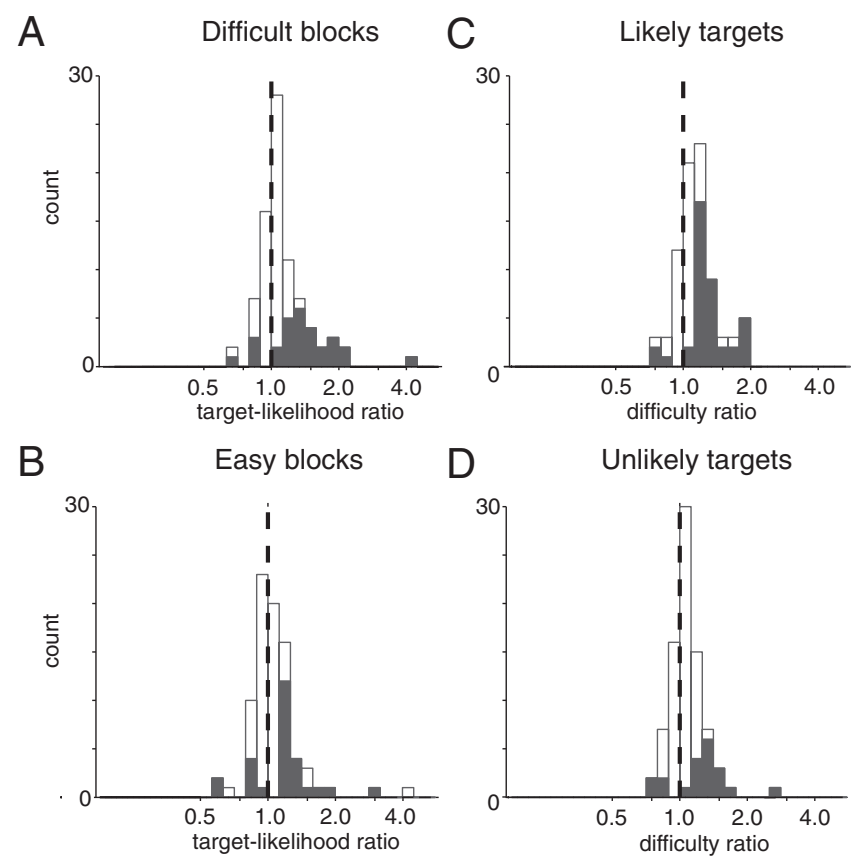

FIG. 4. Target-likelihood and difficulty ratios for Animals 1 and 2. $A$ and $B$ : effect of target likelihood shown as a ratio (likely/unlikely) and plotted on a log scale. Significant effects $(P<0.05)$ are shown in dark gray. $A$ : in difficult blocks, cells responded more to likely targets (Animal 1, 17/51 significant, median increase 9.0\%; Animal 2, 12/32 significant, median increase 4.3\%). B: in easy blocks, the effect of target likelihood was smaller (Animal 1, 21/51 significant, median increase 6.1\%; Animal 2, 6/32 significant, median increase $1.7 \%$ ). $C$ and $D$ : effect of task difficulty is shown as a ratio (difficult/easy) and plotted on a $\log$ scale. Conventions as in $A$ and $B . C$ : increased difficulty caused larger responses to the likely target (Animal 1, 20/51 significant, median increase 10.9\%; Animal 2, 20/31 significant, median increase 18.3\%). $D$ : a more difficult task also increased responses to the unlikely target (Animal 1, 12/51 significant, median increase 5.1\%; Animal 2, 9/32 significant, median increase $7.5 \%$ ). 
interaction between task difficulty and target likelihood that could not be explained by multiplicative scaling $(\mathrm{F}=5.1, P=$ $0.03)$. This interaction also was found for responses to nonpreferred orientations $(\mathrm{F}=8.6, P=0.005)$. To further examine the interaction between task difficulty and target likelihood, we predicted the responses to a likely target in a difficult block from the responses in the other three behavioral conditions. If the difficulty and likelihood effects simply scaled one another, then the ratio of the response to a likely target in a difficult block to an unlikely target in an easy block would be the product of the target-likelihood ratio in the easy block and the difficulty ratio for unlikely stimuli. We found that these predictions underestimated the true size of the effect by a factor of $0.05 \pm 0.01$ on average, which was again a small but significant effect $(P<0.05$ by $t$-test on lognormal data). These results show that the effects of increasing task difficulty are significantly different, but only slightly, from a multiplicative increase on responses to both likely and unlikely targets.

\section{Distractor exclusion}

The neurophysiological data from Animals 1 and 2 suggest that attending more to one part of a visual scene does not require suppressing neuronal responses to stimuli in other parts of the scene. However, there may be cases in which it is beneficial to be able to suppress representations of distracting stimuli. The data from the third monkey, Animal 3, were consistent with this possibility. Animal 3 had previously been trained on a similar task in which multiple distractor stimuli were placed close to the target and responses to orientation changes in the distractors were never rewarded. After retraining on the current task, Animal 3 was able to perform the task well in both easy (94\% correct) and difficult (86\% correct) blocks. Animal 3 showed significant behavioral effects of both task difficulty and target likelihood (Fig. 2). Although the behavior of this animal was qualitatively similar to that of the others, he exhibited a much greater decline in performance for unlikely stimuli as task demands increased, suggesting that he may have been ignoring these stimuli more than the other animals.

\section{Suppression of unlikely target representations associated with distractor exclusion}

Figure 5, $A$ and $B$ shows the cycle average histograms of a neuron from Animal 3 that was strongly modulated by task difficulty. Although there was little effect of target likelihood in the easy blocks, the response to a likely target was increased when the task became more difficult (15\% increase). More strikingly, the mean response to a preferred stimulus that was an unlikely behavioral target was strongly suppressed relative to the easy condition ( $42 \%$ decrease).

Unlike neurons from the previous animals, the 44 neurons recorded from Animal 3 tended to have suppressed responses to unlikely targets in the difficult blocks. Figure 5, $C$ and $D$ shows average responses from all neurons in this subject that were individually significantly modulated by target likelihood in either the easy or difficult trials $(n=25,57 \%$ of neurons; $t$-test, $P<0.05$ with Bonferroni correction for multiple comparisons). The mean driven rate for these neurons in easy trials was 16.0 spikes/s for the likely location and 14.7 spikes/s for the unlikely location. In the difficult condition, the mean driven rate at the likely location rose to 18.7 spikes/s, whereas that for the unlikely location dropped to 11.6 spikes/s. A similar, but attenuated, pattern was seen in the responses of these neurons to the nonpreferred orientation (mean driven rate for easy trials: likely location 6.8 spikes/s, unlikely location 6.8 spikes/s; difficult trials: likely location 8.8 spikes/s, unlikely location 5.2 spikes/s).

Across all the neurons recorded from Animal 3, there was no significant effect (by paired $t$-test, $P>0.5$ ) of target likelihood in the easy blocks, but responses were clearly different for likely and unlikely targets in the difficult blocks $(P<0.001)$. A repeated-measures ANOVA showed no main effect of target likelihood, but a strong effect of task difficulty and a strong interaction between the two.
A

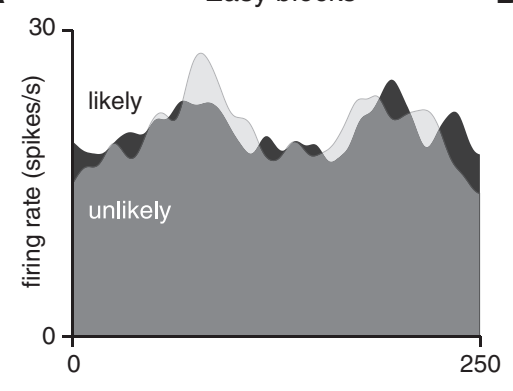

C

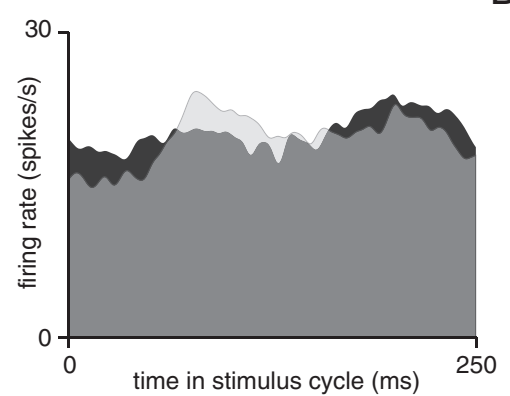

B
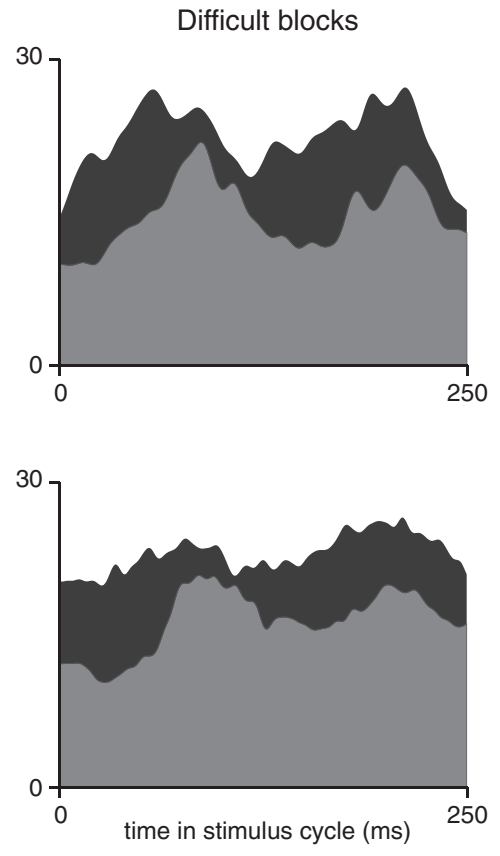

FIG. 5. Effects of load and likelihood on neuronal responses in Animal 3. Same format as Fig. 3. $A$ and $B$ : response histograms from an example cell for its preferred orientation. In the easy trials the average driven rate was 15.8 spikes/s for stimuli at the likely location and 15.3 spikes/s for stimuli at the unlikely location. In difficult trials the average response was $18.1 \mathrm{spikes} / \mathrm{s}$ for the likely location and 8.9 spikes/s for the unlikely location. $C$ and $D$ : population response histograms from the 25 neurons in Animal 3 that were significantly modulated by attention. In the easy trials the average response was 16.0 spikes/s for stimuli at the likely location and 14.7 spikes/s for stimuli at the unlikely location. In difficult trials the average response was 18.7 spikes/s for the likely location and $11.6 \mathrm{spikes} / \mathrm{s}$ for the unlikely location. 
The difference in target-likelihood effects in easy and difficult blocks for Animal 3 is also obvious in the distribution of effects across all cells. Figure $6, A$ and $B$ shows the distribution of target-likelihood ratios in the two conditions. Whereas 28/44 cells showed significant likelihood effects in the difficult blocks, only $8 / 44$ had a significant effect in easy blocks. The median target-likelihood ratio was 1.39 in difficult blocks and 0.99 in easy blocks. Figure 6, $C$ and $D$ shows the effects of difficulty on likely and unlikely target responses. Difficulty effects for likely targets were seen for 12/44 cells and 17/44 cells showed difficulty effects for unlikely targets. Whereas the median difficulty ratio for likely targets was 1.12 , the median ratio for unlikely targets was 0.78 , indicating that the difficulty effects on responses to unlikely targets were primarily suppressive in this animal. There was no correlation between difficulty effects for likely and unlikely targets in our sample of cells, so that the cells that showed enhanced responses to likely targets were not necessarily those showing suppression of responses to unlikely targets. A one-tailed $t$-test of the difficulty ratios for unlikely targets confirmed the suppressive effect $(P<0.001)$.

\section{Vigilance or fixed resource?}

All three animals showed about a $13 \%$ increase in neuronal responses to likely targets as task difficulty increased. This is smaller than the difficulty-related changes reported by Spitzer and colleagues (1988), and may be related to the use of the second behaviorally relevant stimulus in our task, which encouraged some attention to both stimulus locations under all behavioral conditions. In Animal 3, neuronal responses to unlikely targets, instead of increasing slightly with task difficulty, showed a large decrease on average. This suppressive
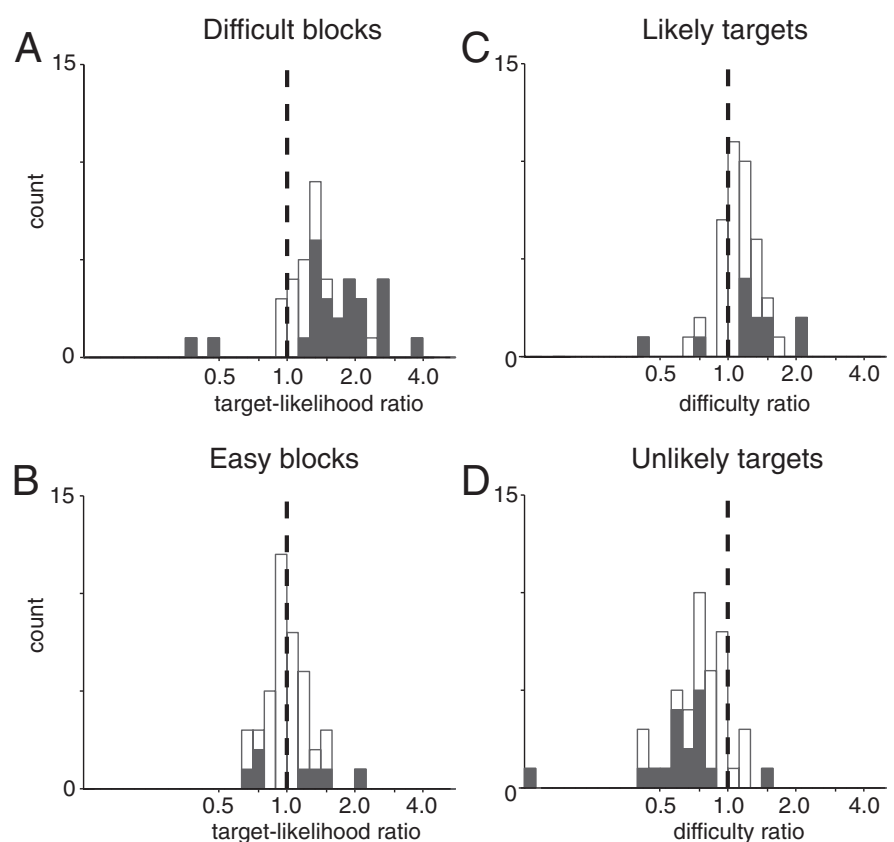

FIG. 6. Target-likelihood and difficulty ratios for Animal 3. Same format as Fig. 4. A: when the task was difficult, there was a strong effect of target likelihood (28/44 significant, median 39\% increase). B: in easy blocks, target likelihood had little effect on responses (8/44 significant, median $1.3 \%$ decrease). $C$ : responses to likely targets are increased in difficult blocks (12/44 significant, median $12.3 \%$ increase). $D$ : responses to unlikely targets are suppressed in difficult blocks (17/44 significant, median $22.2 \%$ decrease). effect of increasing task difficulty on the responses to an unlikely target is consistent with a fixed-resource hypothesis; the decrease in responses would be caused by a withdrawal of response-enhancing attentional resource. The increase in responses to stimuli at the likely location with increasing difficulty would be related to a tighter distribution of the resource around that location. The data from Animals 1 and 2, however, are inconsistent with this explanation and suggest instead an increase in vigilance, with an increase in the amount of resource applied to the task in the difficult mode. Although responses increased at the likely location with increasing difficulty for these animals as well, there was no corresponding decrease in responses to unlikely targets. If the source of the enhancement at the likely location is a greater concentration of resources, then those resources do not seem to have been drawn from the unlikely location. Instead responses to unlikely targets were increased slightly with increasing task difficulty, so that enhancement occurred at both locations.

To examine the extent to which cells from the three animals met the predictions of either the redistribution of a fixed resource or an increase in the amount of resource, we calculated a difficulty index (DI) for each cell and each target likelihood condition. This index, $\mathrm{DI}=\left(\mathrm{R}_{\text {diff }}-\mathrm{R}_{\text {easy }}\right) /\left(\mathrm{R}_{\text {diff }}+\right.$ $R_{\text {easy }}$ ), can take values from -1 to 1 , with 0 indicating that task difficulty had no effect on the response of the neuron, positive values indicating larger responses in the difficult condition, and negative values indicating suppressed responses in the difficult condition. Figure 7 shows the effect of increased task difficulty on the neuronal response to likely targets against its effect on the neuronal response to unlikely targets for all cells in all animals. Because increased difficulty tended to increase responses to a likely stimulus in all animals, most points lie above the $x$-axis. The upper quadrants of the plot are labeled with the hypotheses that best describe the modulation of cells that lie within them. An increase in vigilance would increase neuronal responses regardless of target types, so cells that conformed to this description would be found in the upper right quadrant. Only a few neurons from Animal 3 are found in this quadrant. Resource constraints on spatial attention, on the other hand, should cause responses to unlikely targets to decrease with increasing task difficulty, as resources are withdrawn. Cells whose responses were consistent with this description should fall in the upper left quadrant. Few neurons from Animals 1 and 2 do so. Thus the data from Animals 1 and 2 are consistent with an increase in the neuronal resources applied to the task, and the data from Animal 3 are best explained by the redistribution of a fixed resource (see DISCUSSION).

\section{Behavioral performance with no spatial cue}

The neuronal data from Animals 1 and 2 suggest that increasing task difficulty caused an increase in neuronal firing rates both at and away from the focus of spatial attention. If increases in neuronal response can be taken as an index of the amount of attention, more attention was devoted to both locations, proportional to their established relevance. If this were true, then the influence of task difficulty would not depend on having a spatial cue. To further examine the animals' strategies, we tested behavioral performance in a modified version of the original task. Trials were still performed in easy and difficult blocks, but both stimulus locations were 


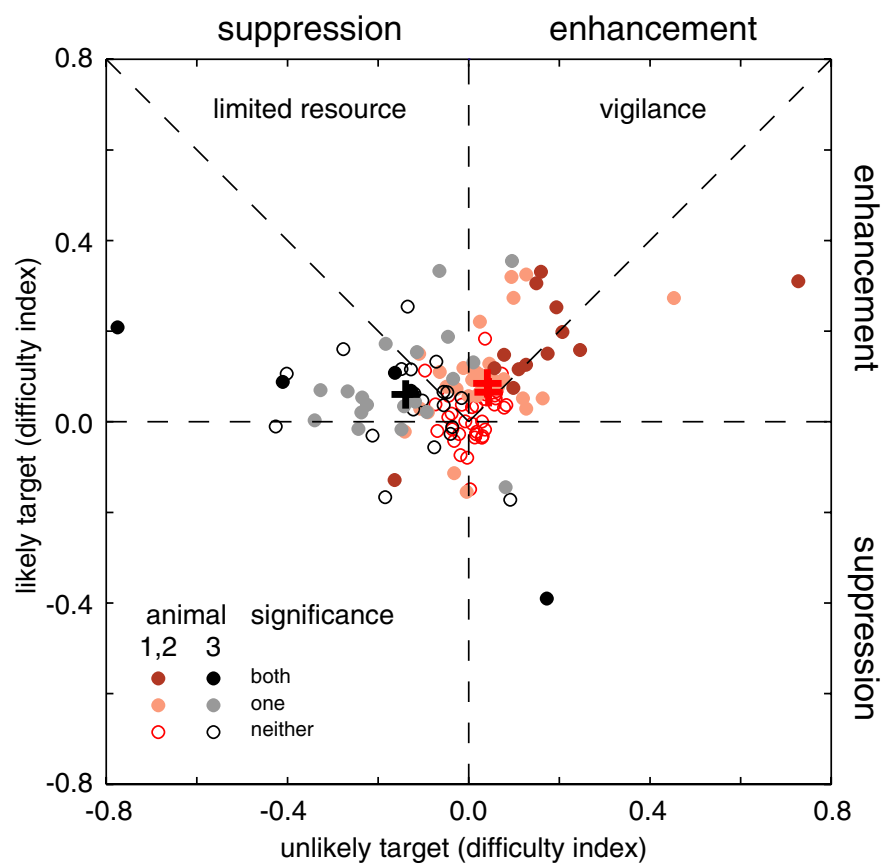

FIG. 7. Comparison of task difficulty effects. This plot shows the effect of increasing difficulty on unlikely ( $x$-axis) and likely ( $y$-axis) target responses. Points above the $x$-axis are cells with enhanced responses to likely targets in the difficult blocks. Points to the right of the $y$-axis represent cells with enhanced responses to unlikely targets in difficult blocks, whereas points to the left represent cells with suppressed responses to unlikely targets. Data from Animals 1 and $2(n=84)$ are shown in red; data from Animal $3(n=44)$ are shown in gray. Cells with significant difficulty effects are filled, with dark fills for cells that had significant effects on both likely and unlikely target responses. Crosses show the mean difficulty indices for each animal (Animal 1: unlikely 0.05 , likely 0.07; Animal 2: unlikely 0.04, likely 0.09; Animal 3: unlikely -0.14 , likely 0.06 ).

equally likely to contain the orientation change. At the beginning of each block, cue stimuli were presented at both stimulus locations. As before, the effect of task difficulty on performance was assessed by measuring accuracy for detection of probe trials, in which the amount of orientation change was intermediate to the two block difficulties and identical regardless of the block in which they occurred.

Figure 8 shows the performance of the animals for probe stimuli in doubly cued trials for easy and difficult blocks. Despite the lack of spatial information, Animals 1 and 2 still showed a significant improvement in their ability to detect small changes in orientation in the difficult blocks, relative to the easy blocks (Animal 1 easy: $64 \pm 3 \%$ correct, difficult: $76 \pm 3 \%$ correct; Animal 2 easy: $67 \pm 2 \%$ correct, difficult: $76 \pm 2 \%$ correct; both $P<0.05, \chi^{2}$ test). These results support the idea that the animals responded to increased difficulty by increasing the amount of attention devoted to the task in a nonspatially specific manner, similar to that propounded by psychophysical studies of vigilance (Sarter et al. 2001).

Unlike the other animals, whose performance on probe trials was improved in difficult blocks in the absence of a spatial cue, Animal 3 did not improve when he could not exclude one of the target locations (easy 67.1\%, difficult 66.4\%; $P>0.9$ ). This is consistent with the idea that this animal responded to increased difficulty by focusing attention more on the likely location when information about target likelihood was available.

\section{Modulation of spontaneous firing}

Functional imaging studies of spatial attention frequently show an increase in blood oxygenation level-dependent (BOLD) signal in visual areas associated with an attended region of space, even in the absence of a stimulus (Kastner et al. 1999; Ress et al. 2000), and evidence from single-unit recordings suggests that this may be associated with an increase in the activity of individual neurons when no stimulus is present (Luck et al. 1997; Williford and Maunsell 2006). We measured our responses relative to baseline firing rate, so we wanted to make sure that spontaneous firing rates were not themselves appreciably altered by our behavioral manipulations. Increases in baseline firing rate could make stimulusinduced responses more difficult to detect, so that larger firing rates might not correspond to enhanced neuronal representation.

Because the spatial location of the likely target and the difficulty level were consistent over long blocks of trials, it seemed possible that spontaneous rates were affected by block type. However, we saw no effect of target likelihood or task difficulty on the spontaneous firing rates in our sample for Animals 1 and 2 (all $P>0.2$, paired $t$-test). Additionally, we calculated the discriminability of the driven response from baseline firing ( $d^{\prime}$ measure; Green and Swets 1966) in each behavioral condition for each cell. We found that discriminability paralleled firing rate (difficult likely $1.86 \pm 0.01$, unlikely $1.67 \pm 0.01$; easy likely $1.68 \pm 0.01$, unlikely $1.66 \pm$ 0.01 ), with significant effects of both task difficulty and target likelihood by repeated-measures ANOVA. Again, discriminability was not decreased and in fact slightly increased for unlikely targets when the task was more difficult.

We also examined the baseline firing rates in different behavioral conditions for Animal 3. In a repeated-measures ANOVA, there was a main effect for target likelihood, no effect of difficulty, and no interaction. Baseline firing rates tended to be higher for unlikely targets $(5.9 \pm 0.1$ and $5.5 \pm$

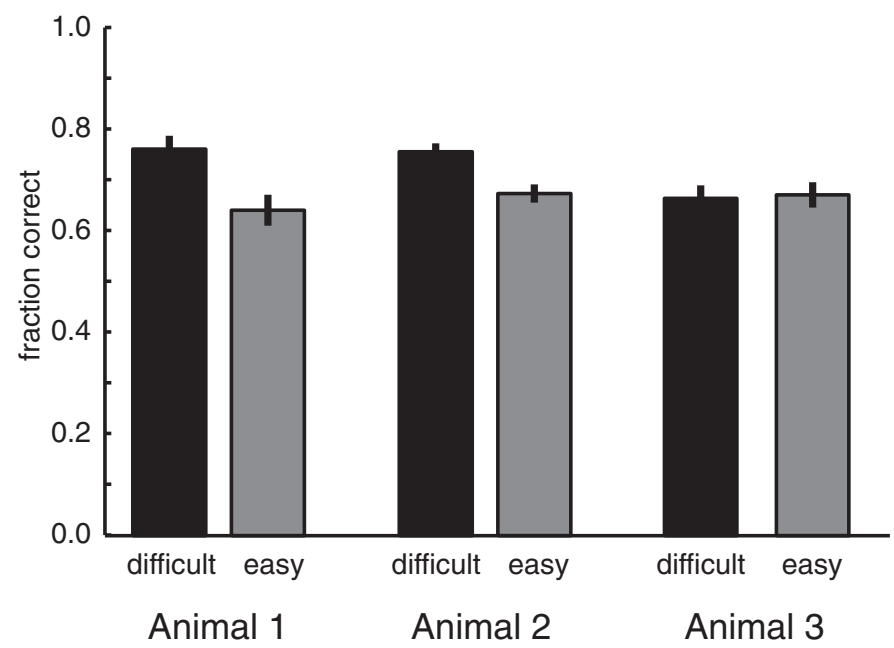

FIG. 8. Behavior in the absence of a likelihood cue. Performance metric as in Fig. 2. Black bars show performance in difficult blocks and gray bars show performance in easy blocks. Of the 2 stimulus locations both were equally likely to contain the orientation change, and animals were not instructed at the beginning of the blocks (Animal 1, $n=508$; Animal 2, $n=669$; Animal 3, $n=696)$. Animals 1 and 2 continued to perform better on more difficult trials, but Animal 3 did not. Task difficulty did not affect any of the animal's relative performance at the 2 stimulus locations ( $\chi^{2}$ test, $\left.P>0.05\right)$. 
0.1 spikes/s in difficult and easy blocks, respectively) than for likely targets $(5.1 \pm 0.1$ and $5.2 \pm 0.1$ spikes/s in difficult and easy blocks). A discriminability analysis showed, again, a main effect of difficulty but not for target likelihood. Difficulty interacted with target likelihood to produce the lowest discriminability for unlikely targets in the difficult task condition (difficult likely $1.69 \pm 0.02$, unlikely $1.30 \pm 0.02$; easy likely $1.40 \pm 0.02$, unlikely $1.46 \pm 0.02$ ). That spontaneous activity was slightly suppressed at likely target locations suggests that in this animal attention may have had an effect of reducing noise in the neuronal representation ( $\mathrm{Lu}$ and Dosher 1998, 2000; Serences et al. 2004).

\section{Eye position differences and eye movements cannot account for observed effects}

Although we attempted to keep visual stimulation the same under all behavioral conditions, it is possible that the animals systematically varied the position of their gaze within the fixation window between conditions, leading to a different retinal position for the stimuli and therefore different neuronal responses (and, potentially, different behavioral performance). We measured the mean eye position separately for the two target likelihood conditions and examined the difference parallel a line joining the two stimuli. Although all of the animals achieved excellent fixation, each had a statistically significant offset as a function of which location was likely to have the target. Animals 1 and 2 had average offsets of $0.01^{\circ}\left(0.003^{\circ}\right.$ $\mathrm{SE})$ and $0.02^{\circ}\left(0.006^{\circ} \mathrm{SE}\right)$ away from the likely location, whereas Animal 3 had an average offset of $0.01^{\circ}\left(0.003^{\circ} \mathrm{SE}\right)$ toward the likely location. All of these offsets were small compared with the spatial frequency of the stimuli, and there is no reason to believe that a small systematic offset in eye position would have a systematic effect on firing rate across the sample of neurons. We therefore believe that the effects of spatial attention and task difficulty that we observed were not the result of differences in eye position between the behavioral conditions.

To look for evidence of systematic slow drifts in eye position during fixation, we measured the cumulative point-topoint distance from initial eye position to the eye position at the end of the trial. Except on trials that contained the relatively large and rapid eye movements that we detected as saccades, these distances were on the order of the intrinsic noise in the eye position signal. We also examined the distribution of eye positions at the end of trials relative to the initial eye position on each trial. These distributions were uniform around the origin for all behavioral conditions in all animals, with a range of $0.5^{\circ}$ in Animal $1,0.9^{\circ}$ in Animal 2 , and $0.9^{\circ}$ in Animal 3 .

\section{I S C U S S I O N}

We examined the effect of task difficulty and target likelihood on neuronal responses in monkey V4. In accordance with a previous study of task difficulty in V4 (Spitzer et al. 1988), we found that increasing task difficulty enhanced responses to an attended stimulus and that this increase correlated with an improved ability to detect small changes in orientation at the attended location.

We also explored how task difficulty affected the representation of relevant stimuli away from the focus of attention, which was not previously examined in single-unit recordings. The results of previous psychophysical studies (Lavie 1995; Lavie and Driver 1996; Lee et al. 1999) and fMRI studies (de Fockert et al. 2001; Pinsk and Kastner 2001) suggest that increasing task difficulty should suppress the processing of stimuli outside the attended location. Although all of the animals in this study exhibited a pattern of behavior consistent with this prediction, neurophysiological analysis revealed that the responses to unlikely stimuli were not suppressed and, in fact, were greater in V4 with increased task difficulty in two of the animals. In a third animal responses to unlikely targets were indeed suppressed when the task was harder. Behavioral tests without a spatial cue to direct attention suggested that the animals used different strategies to cope with increased task difficulty.

Theories of human attention frequently invoke both a fixedcapacity, spatially selective attentional mechanism and a nonspatially specific vigilance mechanism to explain the behavioral effects of attention (Parasuraman 1998). Studies of attention to large or small spatial scales have demonstrated that humans can attend to local or broad areas of visual space voluntarily (Greenwood and Parasuraman 1999; Saarinen 1994, 1995). Monkeys are likely to have the same dichotomy of attention. Although it is possible that the neurophysiological difference between the animals represents some fixed difference in the organization of their nervous systems, it might also be that the balance between spatially selective and nonspatially selective mechanisms is under volitional control and that the animals chose different approaches. If so, neither strategy conferred an absolute behavioral advantage because the average performance for the animals across all conditions was similar. Nevertheless, it is important to consider the significance and possible causes of this difference.

Whereas Animals 1 and 2 never had to ignore more than one distractor, Animal 3 had previously been trained to perform a task that required it to ignore orientation changes in multiple distractors, some very close to the target location. Animal 3 appeared more willing to sacrifice rewards from responses to changes at the unlikely location when the task became more demanding. This may have been because changes at uncued locations had not yielded rewards during his earlier training, even though his recent experience (about $4 \mathrm{mo},>100,000$ trials) specifically encouraged responses to them. It also seems likely that the spatial arrangement or number of distractors in his previous training may have encouraged this animal to adopt a distractor-exclusion strategy. Animals 1 and 2 adopted a strategy in which the representation of an unlikely stimulus remained strong in V4; increasing task difficulty caused an increase in attention in a nonspatially specific manner, leading to improved performance even when spatial attention could not be directed to a single stimulus location. When the spatial cue was present, these animals did use it to bias their attention, although to a lesser extent than Animal 3.

Because the neurophysiological differences we observed correlate with different types of training, it would be very interesting to know whether training can reliably alter the neurophysiological effects of attention. The current experiment was not designed to address whether differences in training or behavioral strategies are associated with different neurophysiological signatures and, although the results are consistent with this notion, they remain inconclusive. Retraining Animals 1 
and 2 on a task that required them to ignore distractors would be unlikely to provide conclusive results. Because the most likely explanation for the differences between the animals is that the initial training of Animal 3 dominated its behavioral strategy, we would similarly predict that the strategies of Animals 1 and 2 would be dominated by their initial training and that their neurophysiology would be unchanged by retraining. Similar neurophysiological differences after retraining would not resolve whether the differences were determined by initial training or unidentified factors. The relationship between training and neurophysiology could be addressed conclusively if additional animals were examined, but power analysis indicates that even if the effect size for training approached five, at least three more animals would need to be trained and recorded to provide a statistically reliable answer (90\% power, $\alpha=$ 0.05). More testing could easily reveal that the effect size is appreciably smaller than this, so that far more animals would be needed to provide sufficient statistical power to draw conclusions about the effects of training. For this reason it would be impractical to attempt to resolve this question with existing methods.

A direct relationship between neuronal responses in V4 and behavioral performance seems plausible for the data from Animal 3, where the population response in each behavioral condition is correlated with the animal's performance in that condition. The data from Animals 1 and 2, however, do not have such a direct relationship to behavior because these animals performed more poorly on unlikely stimuli in the difficult condition, although the neuronal responses to such stimuli strengthened. Previous anatomical and lesion studies have suggested a special role for V4 in the process of selecting relevant visual stimuli (Desimone et al. 1993; De Weerd et al. 1999; Schiller and Lee 1991), and in Animal 3 it appears that, in the difficult condition, the representation of the unattended stimulus is suppressed at or before this stage of processing. The data from Animals 1 and 2, however, show that enhanced neuronal responses do not always translate into improved behavioral performance.

Although difficulty had different effects on the representation of stimuli away from the focus of attention, all three animals were consistent in one respect. For all three the difference in response strength between the high-likelihood and low-likelihood targets increased with increasing difficulty and this was associated with the greater difference in behavioral performance in the difficult condition. This is consistent with the suggestion of Bisley and Goldberg (2003) that behavioral performance for a given target depends on the pattern of activity across populations of neurons and that the relative priority given to different visual stimuli follows the relative activity of the cortical neurons that represent them. They also described that the advantage in behavioral performance for one stimulus was larger when responses to that stimulus were larger relative to another. Their results and ours support the notion that a monotonic function relates relative behavioral performance for two stimuli and the relative strength of neuronal responses to those stimuli. It remains to be seen whether that relative neuronal response predicts relative behavioral performance in cluttered displays that more closely approximate natural viewing conditions. It also remains unclear what weighting rules for neuronal activity across the representation of the image determine the absolute behavioral performance for a given stimulus.

Although the pattern of behavior shown by Animals 1 and 2 was statistically significant and reliable, these animals showed less impairment for detecting changes at the unlikely location in the difficult blocks than did Animal 3. The difference in the physiology between the animals may reflect the degree to which they used the spatial cue to influence the distribution of attention in space; and, whereas the behavior of all three animals was qualitatively the same, strong suppression of behavioral responses to irrelevant stimuli may be generated only by early suppression of sensory representations of those stimuli. Behavioral and neurophysiological differences are commonly seen between different human and animal subjects performing a given task (Britten et al. 1992; Huk and Heeger 2002). Different behavioral strategies may account for some of this variance.

Our results demonstrate that both the distribution of attention between two stimuli and the amount of attention available for a given task are dynamic, depending on the target likelihood at different locations, the attentional load of the task, and on the strategy of the observer. The choice of a strategy for allocating attention may depend on details of task structure, training history, and specific instructions to the observer. One implication of this is that quantitative comparisons of attentional modulations between studies or even task conditions are problematic and should be approached cautiously. Another implication is that the establishment of a veridical amount of attentional modulation for a neuron or a neural structure is not possible. The magnitude of attention effects observed in a given visual area will vary with task design; moreover, even within the same task, different subjects may choose to solve the task in different ways, affecting the observed attentional modulation. These results also imply that experiments designed to examine the interaction of other stimulus or task features with spatial attention must be careful to control for possible changes in task difficulty or subject strategy.

We also suggest that whereas one subject chooses to reallocate attention in space without changing the amount of attention devoted to the task, another may increase the overall amount of attention available for the task by increased vigilance. Attention is clearly not a fixed resource, but can vary on a timescale no longer than minutes. This does not mean that there are no limits on attention, however. There are many ways in which more enhancement of neuronal responses by spatial attention may not result in improved performance. Computational studies (Dayan et al. 2000) have shown that learning of reward contingencies in a model system without resource constraints may result in behavioral patterns like those attributed to fixed-capacity attentional mechanisms. Thus even if there were an unlimited capacity to increase neuronal firing rates there would not necessarily be an unlimited capacity to improve behavioral responses. There may also be other costs, such as metabolic constraints, that influence the decision to increase vigilance or to redistribute attention in space.

\section{A C K N OW LE D G M EN T S}

Thanks to E. P. Cook, J. J. DiCarlo, G. M. Ghose, C. J. McAdams, and T. Yang for helpful comments on the preparation of this manuscript. 


\section{G R A N T S}

This work was supported by Human Frontiers Science Research Program Organization and National Eye Institute Grant R01 EY-05911. J. Maunsell is an Investigator with the Howard Hughes Medical Institute.

\section{REFERENCES}

Bichot NP, Thompson KG, Rao SC, and Schall JD. Reliability of macaque frontal eye field neurons signalling saccade targets during visual search. J Neurosci 21: 713-725, 2001.

Bisley JW and Goldberg ME. Neuronal activity in the lateral intraparietal area and spatial attention. Science 299: 81-86, 2003.

Boudreau CE and Maunsell JHR. Is spatial attention a limited resource for V4 neurons? Soc Neurosci Abstr 27: 574.573, 2001.

Britten KH, Shadlen MN, Newsome WT, and Movshon JA. The analysis of visual motion: a comparison of neuronal and psychophysical performance. J Neurosci 12: 4745-4765, 1992.

Dayan P, Kakade S, and Montague PR. Learning and selective attention. Nat Neurosci Suppl 3: 1218-1223, 2000.

de Fockert JW, Rees G, Frith CD, and Lavie N. The role of working memory in visual selective attention. Science 291: 1803-1806, 2001.

Desimone R, Moran J, Schein SJ, and Mishkin M. A role for the corpus callosum in visual area V4 of the macaque. Vis Neurosci 10: 159-171, 1993.

De Weerd P, Peralta MR 3rd, Desimone R, and Ungerleider LG. Loss of attentional stimulus selection after extrastriate cortical lesions in macaques. Nat Neurosci 2: 753-758, 1999.

Green DM and Swets JA. Signal Detection Theory and Psychophysics. New York: Wiley. 1966.

Greenwood PM and Parasuraman R. Scale of attentional focus in visual search. Percept Psychophys 61: 837-859, 1999.

Hasegawa RP, Blitz AM, and Goldberg ME. Neurons in monkey prefrontal cortex whose activity tracks the progress of a three-step self-ordered task. J Neurophysiol 92: 1524-1535, 2004.

Huk AC and Heeger DJ. Pattern-motion responses in human visual cortex. Nat Neurosci 5: 72-75, 2002.

Judge SJ, Richmond BJ, and Chu FC. Implantation of magnetic search coils for measurement of eye position: an improved method. Vision Res 20: 535-538, 1980.

Kastner S, Pinsk MA, De Weerd P, Desimone R, and Ungerleider LG. Increased activity in human visual cortex during directed attention in the absence of visual stimulation. Neuron 22: 751-761, 1999.

LaBerge D, Brown V, Carter M, Bash D, and Hartley A. Reducing the effects of adjacent distractors by narrowing attention. J Exp Psychol Hum Percept Perform 17: 65-76, 1991.

Lavie N. Perceptual load as a necessary condition for selective attention. $J$ Exp Psychol Hum Percept Perform 21: 451-468, 1995.

Lavie $\mathbf{N}$ and Cox $\mathbf{S}$. On the efficiency of visual selective attention: efficient visual search leads to inefficient distractor rejection. Psychol Sci 8: 395-398, 1997.

Lavie $\mathbf{N}$ and Driver J. On the spatial extent of attention in object-based visual selection. Percept Psychophys 58: 1238-1251, 1996.

Lavie N and Tsal Y. Perceptual load as a major determinant of the locus of selection in visual attention. Percept Psychophys 56: 183-197, 1994.

Lee DK, Itti L, Koch C, and Braun J. Attention activates winner-take-all competition among visual filters. Nat Neurosci 2: 375-381, 1999.

Lee DK, Koch C, and Braun J. Spatial vision thresholds in the near absence of attention. Vision Res 37: 2409-2418, 1997.
Lu ZL and Dosher BA. External noise distinguishes attention mechanisms. Vision Res 38: 1183-1198, 1998.

Lu ZL and Dosher BA. Spatial attention: different mechanisms for central and peripheral temporal precues. J Exp Psychol Hum Percept Perform 26: 1534-1548, 2000.

Luck SJ, Chelazzi L, Hillyard SA, and Desimone R. Neural mechanisms of spatial selective attention in areas V1, V2, and V4 of macaque visual cortex. J Neurophysiol 77: 24-42, 1997.

McAdams CJ and Maunsell JHR. Effects of attention on orientation-tuning functions of single neurons in macaque cortical area V4. J Neurosci 19: 431-441, 1999.

Motter BC. Focal attention produces spatially selective processing in visual cortical areas V1, V2, and V4 in the presence of competing stimuli. J Neurophysiol 70: 909-919, 1993.

Parasuraman R. Sustained attention in detection and discrimination. In: Varieties of Attention, edited by Parasuraman R and Davies DR. New York: Academic Press, 1984, p. 243-270.

Parasuraman R. The attentive brain: issues and prospects. In: The Attentive Brain, edited by Parasuraman R. Cambridge, MA: MIT Press, 1998, p. 3-17.

Parasuraman R, Warm JS, and See JE. Brain systems of vigilance. In: The Attentive Brain, edited by Parasuraman R. Cambridge, MA: MIT Press, 1998, p. 221-256.

Pinsk MA and Kastner S. Selective attention and task difficulty: effects on target and distracter stimuli. Soc Neurosci Abstr 27: 574.574, 2001.

Rees G, Frith CD, and Lavie N. Modulating irrelevant motion perception by varying attentional load in an unrelated task. Science 278: 1616-1619, 1997.

Ress D, Backus BT, and Heeger DJ. Activity in primary visual cortex predicts performance in a visual detection task. Nat Neurosci 3: 940-945, 2000.

Robinson DA. A method of measuring eye movements using a scleral search coil in a magnetic field. IEEE Trans Biomed Eng 101: 131-145, 1963.

Saarinen J. Visual search for global and local stimulus features. Perception 23: 237-243, 1994

Saarinen J. Visual search at different spatial scales. Scand J Psychol 36: 1-9, 1995.

Sade A and Spitzer H. The effects of attentional spread and attentional effort on orientation discrimination. Spat Vis 11: 367-383, 1998.

Sarter M, Givens B, and Bruno JP. The cognitive neuroscience of sustained attention: where top-down meets bottom-up. Brain Res Brain Res Rev 35: $146-160,2001$.

Schiller PH and Lee K. The role of the primate extrastriate area V4 in vision. Science 251: 1251-1253, 1991.

Serences JT, Yantis S, Culberson A, and Awh E. Preparatory activity in visual cortex indexes distractor suppression during covert spatial orienting. J Neurophysiol 92: 3538-3545, 2004.

Spitzer H, Desimone R, and Moran J. Increased attention enhances both behavioral and neuronal performance. Science 240: 338-340, 1988.

Spitzer $\mathbf{H}$ and Richmond BJ. Task difficulty: ignoring, attending to, and discriminating a visual stimulus yield progressively more activity in inferior temporal neurons. Exp Brain Res 83: 340-348, 1991.

Urbach D and Spitzer H. Attentional effort modulated by task difficulty. Vision Res 35: 2169-2177, 1995.

Williford T and Maunsell JHR. Effect of spatial attention on contrast response functions in macaque area V4. J Neurophysiol 96: 40-54, 2006.

Zar JH. Biostatistical Analysis. Upper Saddle River, NJ: Prentice-Hall, 1999. 